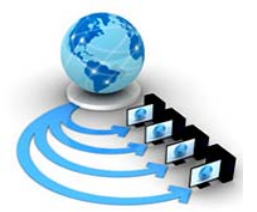

Volume 10, No. 6, November-December 2019

\title{
STUDY ON TRIBOLOGICAL CHARACTERISTICS OF HOISTING EQUIPMENT BRAKE SYSTEM
}

\author{
Sen $\mathrm{ZHANG}^{1}$, Aiqin HUANG ${ }^{1}$, Pehai LIU ${ }^{2}$ \\ ${ }^{1}$ Binzhou University, ${ }^{2}$ Binzhou Bohai piston Co., Ltd \\ Binzhou, China
}

\begin{abstract}
In industrial production, there are strict requirements for the braking effect of heavy transport equipment such as elevator and belt conveyor. The working capacity and reliability of the brake are the basis for ensuring efficient production. Based on the friction and wear test, taking the average friction coefficient and stable wear rate as the key parameters to measure the reliability and stability of the brake, the friction and wear characteristics of the hydraulic disc brake of the hoist under different working conditions are analyzed and studied. The results shows that the average friction coefficient and stable wear rate of the brake fluctuate less with the change of brake pressure in the middle speed range. At this time, the adhesive and matrix fiber in the friction pad have not been decomposed and modified, and the strength and shape of the surface micro convex structure are relatively moderate, so the braking reliability is in good condition.
\end{abstract}

Keywords: computer simulation, brake, metallography, wear, friction

\section{INTRODUCTION}

In the contemporary industrial production, the elevator is an indispensable transportation equipment in many industries and fields [1], which undertakes the transportation of personnel, materials and equipment, and is an important hub associated with different processes [2]. At present, with the development of production technology, domestic industrial production tends to be safe and efficient year by year [3], which puts forward higher and stricter requirements for the working performance of the hoist. As one of the most important parts of the hoist, the hydraulic brake directly determines whether the hoist can decelerate, stop and other tasks can be successfully completed, and also affects the safety of the hoisting work. According to incomplete statistics, the safety accidents caused by the instability or failure of the hydraulic brake account for more than $60 \%$ of the total safety accidents of the hoist, which not only leads to casualties of the staff, but also losses a lot of capital and property.

At present, there are mainly two types of hydraulic brake for the hoist: brake shoe type and disc type. Compared with the brake shoe brake, the disc brake appears later, but its application rate is higher because of its large effective contact area of friction pair, good friction stability, stronger adaptability to harsh environment such as humidity and dust [4]. In addition, the new type of hydraulic disc brake derivative air-cooled groove structure (also known as air-cooled disc brake) further increases the contact area between the brake disc and air, and significantly improves its convection heat transfer ability. In order to get the reliability evaluation of the performance of the actuator of this type of lifting mechanism, it is necessary to study its friction and wear characteristics in depth. Therefore, the friction and wear characteristics of the brake are tested based on the link $3900 \mathrm{NVH}$ test machine, and the worn surface morphology of the friction pad is analyzed by tm3000 SEM.

\section{TRIBOLOGICAL PERFORMANCE TEST OF BRAKE}

\section{A. Analysis of friction braking mechanism}

At present, there are many kinds of hoist disc brakes in the market, and the representative ones are ts215 brake, whose structure is shown in Fig.1. The friction pair of the brake consists of two groups of left and right symmetrical friction pads and brake discs. The friction type is typical dry friction. According to the tribological theory, the braking force between the friction pad and the brake disc comes from three kinds of functions.

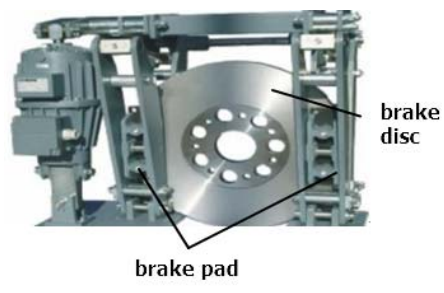

Fig.1Disc brake structure

(1) The brake disc material of this type of brake is HT250, and the friction pad material is organic nonmetal, which has the property of fireproof flower [5]. When the lifting mechanism actuator works, the micro convex structure on the surface of the friction pair will hinder the movement and form the mechanical deformation resistance. This deformation resistance is not completely mechanical dominated, but also related to the intermolecular forces [6].

(2) The mechanism of adhesion is similar to that of cold welding, mainly due to the plastic deformation and adhesion point of the micro convex structure under the extrusion, which has a direct impact on the real contact area between the friction pairs. When the hoist brake is decelerated, the brake is in the dynamic sliding friction state.

(3) Due to the poor working environment of the hoist, the friction pair surface of the brake will inevitably fall or fall off some small hard particles, which will produce furrow effect on the friction pair surface under the brake pressure of the friction pad. 


\section{B. Brake performance test plan}

The parameters of the hoist, such as lifting quality, running speed, braking pressure and temperature, have a certain impact on the tribological characteristics of the brake. Therefore, link3900 NVH bench test machine (as shown in Fig.2) is proposed to test the tribological parameters under different working conditions. The test machine has an inertia flywheel, which can simulate the inertia of the hoist in the lifting process, and a temperature controller is set in the operation box, which can set the ambient temperature. In addition, pressure sensor and speed sensor are integrated in the test machine. An additional thermocouple is installed on the surface of the brake disc to obtain the transient brake temperature in real time.

Set the upper computer software program to maintain the constant pressure (brake pressure) and constant speed (brake disc speed) conditions. In the process from separation to contact, there will be three stages of friction change.

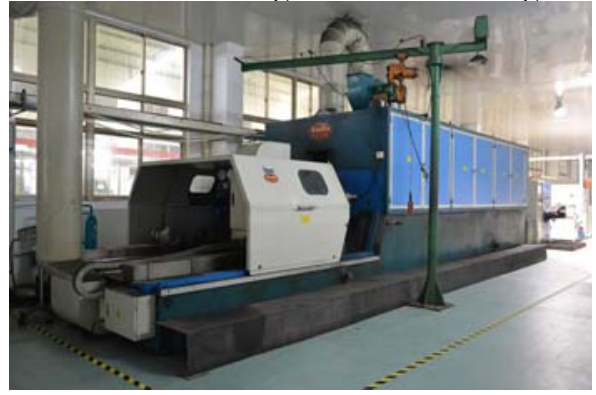

Fig.2 Structure of bench test machine

\section{Analysis of factors influencing tribological properties}

Brake pressure is the most direct and effective factor that affects the brake distance of hoist. In order to study the effect of brake pressure on friction coefficient, constant temperature test conditions $\left(100{ }^{\circ} \mathrm{C}\right)$ were set, and the average friction coefficient under different brake pressure conditions was tested. In addition, according to the working parameters of the hoist, the hoisting speed gear includes: low speed gear (5m / min), medium speed gear $(10 \mathrm{~m} / \mathrm{min})$ and high speed gear (30m / min), which can be adjusted infinitely. For this reason, the test is carried out under six different lifting speed conditions (conversion with the spindle speed), and the results are shown in Fig.3. It can be seen that with the increase of brake pressure, the friction coefficient of the brake shows a downward trend. When the brake pressure is fixed, the friction coefficient of low speed gear is significantly higher than that of medium and high speed gear, and the average friction coefficient of $5 \mathrm{~m} / \mathrm{min}$ lifting speed is stable between 0.51 and 0.68 . According to the above analysis conclusion, it is not reliable and advisable to ensure the braking stability of the hoist only by increasing the braking pressure.

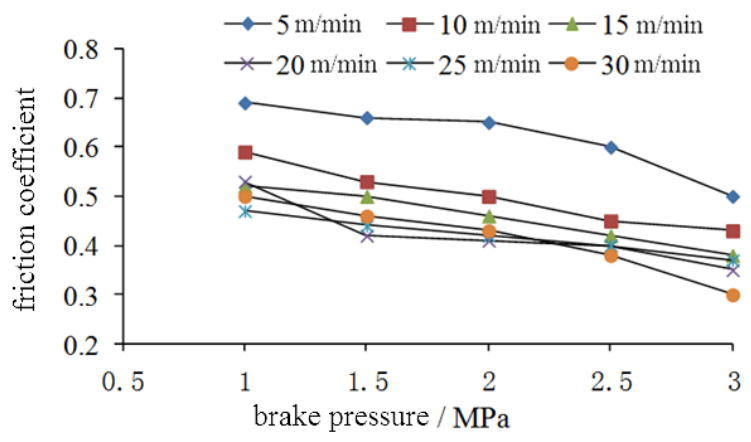

Fig.3 Friction coefficient changes with brake pressure
With the acceleration of industrial production efficiency, the operation speed of the hoist under rated working conditions tends to increase. According to the law of dynamics, the kinetic energy of the hoist is directly related to the lifting speed, and the brake will consume its mechanical energy through friction, so the lifting speed determines the energy input of the brake. In addition, the lifting speed has a certain influence on the friction coefficient. By studying the influence law, it can provide an important basis for the intelligent adjustment of the lifting speed. Similarly, the constant temperature $\left(100{ }^{\circ} \mathrm{C}\right)$ and constant pressure test conditions are set, and the braking condition with constant speed drop is taken as the analysis object. The change of average friction coefficient under different lifting speed conditions is shown in Figure 4. It can be seen that the average friction coefficient tends to decrease with the increase of lifting speed. In the middle speed and high speed gear, the friction coefficient is relatively less affected by the lifting speed, especially in the lifting condition of $15 \sim 25 \mathrm{~m}$ / min, the friction stability is good.

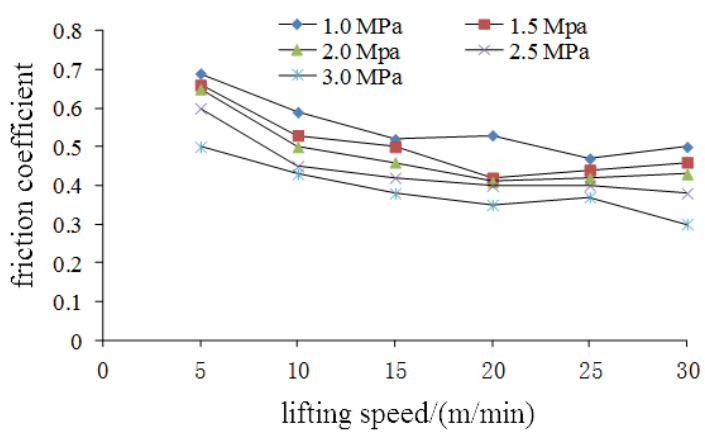

Fig.4 Friction coefficient changes with lifting speed

\section{STUDY ON WEAR CHARACTERISTICS OF BRAKE}

\section{A. Wear mechanism analysis}

As one of the most important factors affecting the reliability of braking, wear is inevitable. After long-term wear, the thickness of the friction pad will obviously become thinner, which has a very adverse effect on the application of braking pressure, and the friction coefficient cannot be kept stable [7]. In essence, wear is a complex micro dynamic process of materials, not an inherent property of materials. The evaluation of wear characteristics can be based on macro and micro aspects. The effect of braking conditions on wear has always been a hot spot in tribology research. Through this research, the effective working life of the brake can be accurately predicted to ensure the safety and reliability of the brake. Generally speaking, there are four wear modes of brake friction pad: adhesive wear, cutting wear, abrasive wear and fatigue wear.

For the friction pad of non-metallic materials, there are often more resin based materials in it. Its strength is significantly reduced after friction heat generation, and it is easy to appear adhesion phenomenon under the action of braking pressure, resulting in adhesion wear. In addition, adhesive wear is also related to the surface tension of the friction pair. Because the hardness of the two surfaces of the brake friction pair is quite different, the hard micro convex structure of the brake disc surface will produce cutting wear on the friction disc surface. Abrasive wear is similar to furrow, the particles falling off or outside will scratch the surface of friction pair, resulting in abrasive wear. The probability of abrasive wear in dust production is high. 


\section{B. Wear rate test}

Wear rate, as a key parameter to measure the macro wear characteristics of the hoist actuator, can effectively reflect the wear resistance of the friction pad. The link $3900 \mathrm{NVH}$ tester used in this paper has a standard wear test module, which can be used to test different brake pressure and lifting speed conditions, and calculate the corresponding wear rate of friction pad. According to the wear test standard of metal material, the friction pad is cut and polished into standard block sample, and the volume wear is taken as the calculation amount of wear rate. Through a single test, it can be seen that the wear amount changes with time in three stages. The initial stage is running in period, the wear rate increases rapidly, and the curvature radius of the micro convex structure on the surface of the friction pair increases. The middle stage is a stable period, and the wear rate basically keeps in a small range. In the last stage, the wear rate increases rapidly again. At this time, the surface temperature of the friction pair is higher and the mechanical properties are significantly reduced.

In order to study the wear resistance of friction pad in normal operation, the wear rate in stable period is taken as the evaluation parameter. In addition, after each friction and before measurement, it is necessary to adjust the friction pad to room temperature and maintain it for a period of time through the thermostat. Finally, it can be concluded that under different lifting speed conditions, the wear rate in stable period changes with braking pressure, as shown in Fig.5. It can be seen that under the condition of low speed and high speed, the wear rate during the stable period is relatively large, and shows a very significant fluctuation with the change of braking pressure. The wear rate in the stable period is low and relatively stable at medium speed. There is no direct relationship between wear rate and brake pressure in stable period.

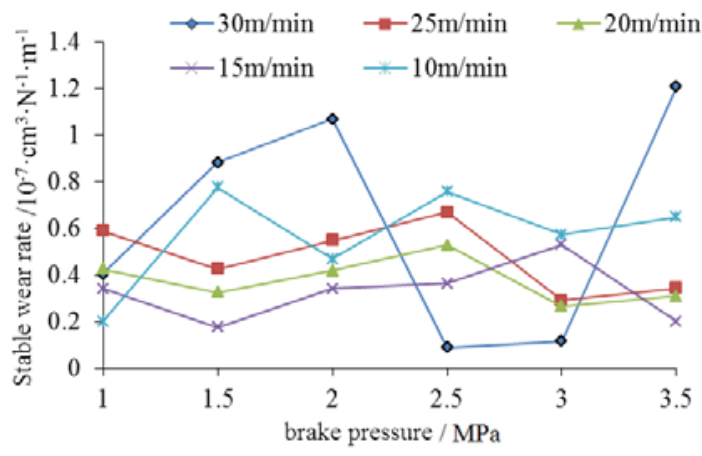

Fig.5 Change of stable wear rate with brake pressure

\section{Wear characteristics analysis}

According to the test results of wear test, it can be seen that the wear rate of the actuator in stable period does not increase continuously with the increase of brake pressure, which is obviously different from the conventional semi metal friction pad. This is because with the increase of braking pressure, the regeneration rate of the micro convex structure on the surface of non-metallic friction pad is higher. However, the yield strength of these micro convex structures is smaller, and they are more prone to plastic deformation, weakening the adhesion and cutting effect, so the difference of wear effect under medium speed condition is smaller.

When the hoist is braking at low speed, the value of stable wear rate increases and decreases with the increase of brake pressure, and the wear resistance shows certain instability. This is due to the fact that under the condition of lower lifting speed, the brake disc speed is lower, and the temperature rise rate of friction pad in unit time is smaller. A small temperature rise rate is not conducive to the formation of new contact spots, but will maintain the strength of the original micro convex structure (at this time, the wear mode is mainly the cutting effect). At the same time, the contact pressure between the friction pairs also has an important influence on the formation of contact spots, especially on the binder (NBR modified phenolic resin) material. It can be seen from the above conclusions that the fluctuation of stable wear rate is the result of the joint action of braking pressure and lifting speed.

When the brake disc rotates at high speed, the heat flux density of the friction pair surface is large, and the binder will soften obviously under the action of large heat load. As the brake pressure continues to increase, the temperature of the friction pad surface will be higher. The softened binder will precipitate lubricating substances, greatly weakening the adhesion and cutting effect, so the wear rate will be reduced. Under the action of continuously increasing brake pressure, the lubricating material will fall off, so the wear rate will increase sharply again after being maintained.

\section{Analysis of wear morphology of friction pad surface}

After the wear test, the standard sample of the friction pad is placed under the TM 3000 scanning electron microscope for observation. Under the condition of $2 \mathrm{MPa}$ brake pressure, the surface wear morphology under the conditions of low speed gear (5m / $\mathrm{min})$, medium speed gear (10m / min) and high speed gear (30M / min) is shown in Fig.6. As shown in Fig. 6 (a), it is the micro morphology under low-speed friction condition. It can be seen that the main components of the surface are matrix fiber, binder and filler. Under the action of friction load and thermal load, the matrix fiber has appeared gray and bright wear marks, but the proportion of granular wear marks is relatively small. As shown in Fig. 6 (b), it is the micro morphology under the medium speed friction condition. It can be seen that the morphology of the friction surface has changed significantly compared with the low speed condition. The binder has been thermally decomposed and modified, and the number of gray and bright particles has increased, but the wear on the surface is relatively uniform, and the plastic flow of the binder has certain directionality. At this time, the friction pair has good friction. Rub effect. As shown in Fig. 6 (c), it is the micro morphology under the condition of medium speed friction. It can be seen that the number of granular wear marks is reduced, and the matrix fiber has appeared irregular decomposition. Although it is not completely dispersed under the action of the binder, the binder also has a certain plastic flow. This stage is the period of friction mutation, and the wear is very severe.

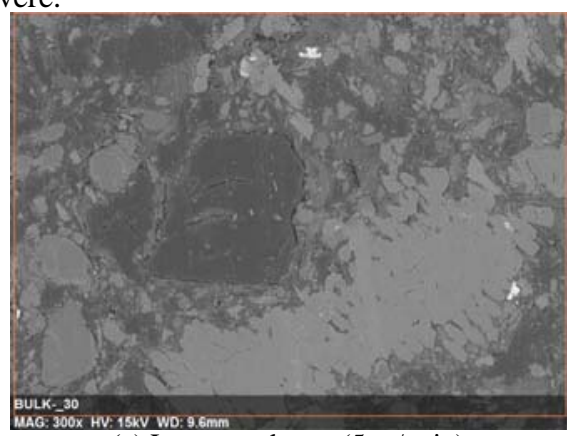

(a) Low speed gear ( $5 \mathrm{~m} / \mathrm{min})$ 


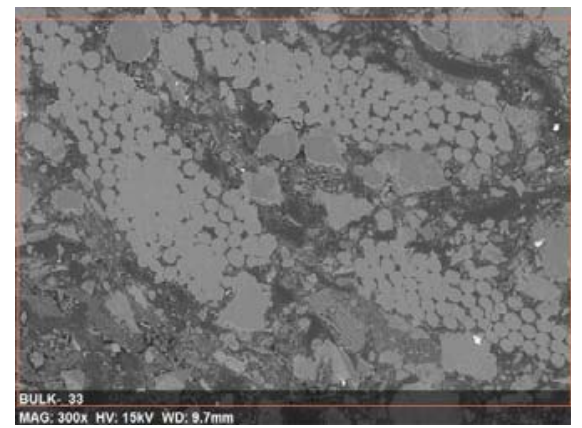

(b) Medium speed gear $(10 \mathrm{~m} / \mathrm{min})$

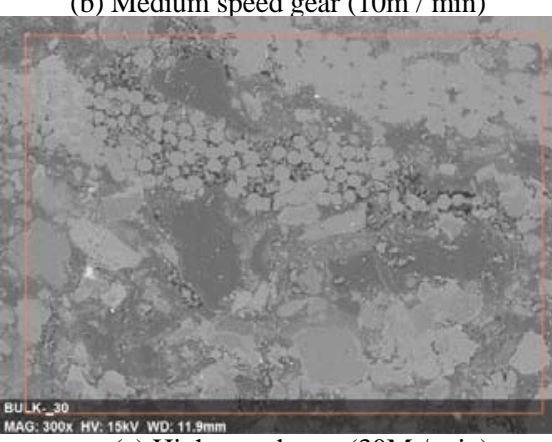

(c) High speed gear (30M / min)

Fig.6 Surface morphology of friction pad

\section{CONCLUSION}

At the initial braking stage, the friction increases continuously, but the growth rate changes from fast to slow. During the braking adjustment, with the contact state maintained, the friction force shows a slight decrease trend at this stage, which is due to the adhesion effect, tends to be significant. At this time, although the real contact area increases, the aggregation speed of contact spots is greater than the generation rate of new contact points, which makes the number of contact spots decrease and the friction coefficient decrease. The duration of the brake adjustment is approximately $0.68 \mathrm{~s}$. When braking stably, the friction coefficient shows a dynamic and stable state, and the value of friction keeps at a certain value and fluctuates in a small range.

\section{REFERENCES}

[1] H. Xiuhua. "Economic evaluation and uncertainty analysis of coal mining plan," Computer Simulation, vol.32, pp.206-209, October 2015.

[2] L. Liu-min, H. Guiyun. "Application of frequency control technology in mine shaft hoist," Computer Simulation, vol.33, pp. 234-237, August 2016.

[3] L. Hongsheng. "Fault analysis and reliability simulation of deep well hoisting wire rope," Computer Simulation, vol. 31, pp. 407-411, January 2014.

[4] L. Qiaohua. "Design of safety monitoring device for disc brake system of hoist based on PLC," Journal of Automotive Engineering, vol. 01, pp. 464-468, April 2014.

[5] Q. Canwen. "Braking simulation of vehicles on opposite pavement," Agricultural Equipment And Vehicle Engineering, vol.3, pp. 45-47, June 2018.

[6] Z. Zhiguo, P. Yugang. "Safety regulation and test of friction spark of metal material blade of coal mine ventilator," Journal of Systems Simulation, vol.24, pp.208-215, July 2015.

[7] W. Kesheng, Z. Leiqiang. "olecular dynamics simulation of dry friction damper with coating," Computer Simulation, vol.28, pp.342-347, April 2013. 